\title{
Functional Decline Status for the Development of Aspiration Pneumonia in Elderly Patients with Femoral Neck and Trochanteric Fractures
}

\author{
Takuro Morita ${ }^{4 *}$, Toshihiro Higashikawa ${ }^{1 *}$, Manabu Moriyama², Hiromi Inujima ${ }^{3}$, Masahiro Hangyou ${ }^{3}$, \\ Kimiko Usuda ${ }^{3}$, Shigeto Morimoto ${ }^{4}$, Masashi Okuro ${ }^{4}$, Tadami Matsumoto ${ }^{5}$, Kenji Shigemoto ${ }^{6}$, Kenichi \\ Goshima $^{6}$, Tsugiyasu Kanda ${ }^{7}$, Daisuke Usuda ${ }^{7}$, Shigeki Takashima ${ }^{7}$ and Takeshi Sawaguchi ${ }^{6}$ \\ ${ }^{1}$ Department of Geriatric Medicine, Kanazawa Medical University Himi Municipal Hospital, Japan \\ ${ }^{2}$ Department of Urology, Kanazawa Medical University Himi Municipal Hospital, Japan \\ ${ }^{3}$ Toyama Municipal Hospital, Japan \\ ${ }^{4}$ Department of Geriatric Medicine, Kanazawa Medical University, Japan \\ ${ }^{5}$ Department of Orthopedic Medicine, Kanazawa Medical University, Japan \\ ${ }^{6}$ Department of Orthopedics and Joint Reconstructive Surgery, Toyama Municipal Hospital, Japan \\ ${ }^{7}$ Kanazawa Medical University, Japan
}

\begin{abstract}
Aspiration pneumonia (AP) has been recognized as one of the most common postoperative complications after hip surgery in elderly. The objective of the present study was to evaluate risk for postoperative complications of AP in elderly patients with femoral neck fractures. We recruited 221 female patients (age $84.9 \pm 7.1$ years) with a history of hip surgery carried out at Toyama Municipal Hospital. AP occurred in 11 out of 276 cases (3.99\%). Fisher's exact test have found significant differences in gender, serum albumin level, cognitive impairment, and history of osteoporosis, between AP and non-AP groups. Subsequently multiple logistic regression analysis was conducted to investigate the risk factors for AP, including age, body mass index (BMI), serum albumin, cognitive impairment, and activities of daily living (ADL). Univariate odds ratio showed significant differences in age and cognitive disorder, although adjusted odds ratio of these variables showed no significant association with AP incidence. This study suggested that serum albumin and cognitive function seemed to be the weak risk factors for AP but were necessary to assess under adjustment of confounding factors, including age, gender and BMI. Taking account of cognitive functional assistance seemed to be important for the postoperative management of AP, especially in elderly patients receiving surgery of femoral neck and trochanteric fractures.
\end{abstract}

KEYWORDS: Elderly; Hip fractures; Aspiration pneumonia; Age; Cognitive impairment

ABBREVIATIONS: : AP: Aspiration Pneumonia; BMI: Body Mass Index; ADL: Activities of Daily Living

Quick Response Code:

Address for correspondence: Takuro Morita, Toshihiro Higashikawa, MD, PhD, Department of Geriatric Medicine, Himi Municipal Hospital, Japan

Received: October 13, $2020 \quad$ Published: October 28, 2020

How to cite this article: Toshihiro H, Takuro M, Manabu M, Hiromi I, Masahiro H, Kimiko U, Shigeto M, Masashi O, Tadami M, Kenji S, Kenichi G, Takeshi S, Daisuke U, Shigeki T, Tsugiyasu K. Functional Decline Status for the Development of Aspiration Pneumonia in Elderly Patients with Femoral Neck and Trochanteric Fractures. 2020 - 2(5) OAJBS.ID.000230. DOI: 10.38125/ OAJBS.000230 


\section{INTRODUCTION}

The incidence of hip fracture in the elderly has been increased rapidly from 1.7 million in 1990 to an estimated 6.3 million in 2050 worldwide [1]. Approximately one third of these patients die within the first year [2]. A nationwide survey of hip fractures by the Japanese Orthopaedic Association (JOA) from 1998 to 2008 found a drastic increase in incidence [3]. As a consequence, geriatric and orthopedic collaboration (orthogeriatrics) has been organized in different ways in Japan. Such collaboration has been conducted in Toyama city, which locates in the middle district in Toyama Prefecture which has an area of approximately 1,200 square kilometers and has about four hundred thousand residents [4]. The proportion of elderly people aged 65 and over to all the residents in Toyama city is over $30 \%$. Toyama Municipal Hospital is a core hospital in Toyama Prefecture which has approximately 600 beds. In the hospital, an Elderly Bone Femoral Neck and Trochanteric Fracture Center has been installed with advanced technology and dedicated staffs to conduct surgery in patients with acute phase femoral neck and trochanteric fracture. Co-management of hip fracture patients by orthopedic surgeons and geriatricians is suggested to be effective to reduce length of hospital stay without negatively affecting major patient outcomes [2]. On this account, multidisciplinary cooperation could be effective as conducted in Toyama Municipal Hospital.

The cause of mortality in elderly people with hip fractures mostly attributed to postoperative complications, such as wound infections, pneumonia, deep venous thromboses, respiratory and urinary infections and cardiovascular events [5-12]. The consequences of hip fractures in elderly persons also include a deterioration in functional capacity to perform activities that enable independent living $[13,14]$.

Among these patients, cases of complicated pneumonia are frequently encountered, but there are few reports investigated complications of pneumonia $[14,15]$. Therefore, the present study was retrospectively investigated on the cases suffering aspiration pneumonia which was hospitalized for femoral fracture and received operation and rehabilitation. In addition, we examined risk factors for pneumonia, particularly focusing on risk factors for aspiration pneumonia to identify some measures to raise the patient's QOL.

\section{MATERIALS AND METHODS}

This retrospective study was carried out under approval (2018-06) of the Clinical Research Ethics Committee of Kanazawa Medical University. Demographic data were collected by physicians, specialist nurses, pharmacists, and medical affairs. The existence of the aspiration pneumonia was also examined in the same division. Body mass index (BMI) was calculated as weight $(\mathrm{kg})$ divided by the square of height $\left(\mathrm{m}^{2}\right)$. Waiting time (day) from hospitalization to entering the operating room was also calculated on each case.

The aspiration pneumonia was diagnosed by postoperative chest X-ray by radiologists. The cases were defined as positive of aspiration pneumonia (AP positive) and others were defined as negative of aspiration pneumonia (AP negative). Serum albumin concentration was measured and the value itself was used for the following statistical analysis as continuous variables. In addition, the data were divided into high albumin $(\geq 3.5 \mathrm{~g} / \mathrm{dL})$ and low albumin $(<3.5 \mathrm{~g} / \mathrm{dL})$ groups that were used for the following analysis as dichotomous variables.
Cognitive disorders were diagnosed by neuropsychiatrists. Degree of independence in everyday life on each case was assessed by a conventional ADL scale, which is classified by the four groups (J, A, B, C). The group J is mostly assistance-free, the group A is semi-bedridden mostly active during daytime, the group $B$ is bedridden requiring assistance for daily activities, and the group $C$ is bedridden requires assistance in bed $[16,17]$. We divided them into two groups, J A (assistance non-required), and B C (assistancerequired) group.

Data of history of hypertension, diabetes mellitus, circulatory disease, respiratory disease, renal disease, bone fracture and osteoporosis were collected from medical records of the cases. The present study consists of continuous data, such as age, BMI, waiting time, serum albumin concentration, which were statistically evaluated by mean, standard deviation and Student's t-test. The present study also consists of dichotomous data, such as categorized serum albumin concentration, cognitive disorder, ADL, and history of diseases, which were statistically evaluated by Fisher's exact test.

Logistic regression analyses were performed using dichotomous $\mathrm{AP}$ positive and AP negative as dependent variables, and age, BMI, serum albumin, cognitive disorder and ADL as independent variables. All statistical analyses were two-tailed, $\mathrm{p}<0.05$ regarded as significant, including Student's t-test, chi-square test and logistic regression analyses performed using EZR.

\section{RESULTS}

Initially 321 patients were admitted to the Toyama Municipal Hospital from January $1^{\text {st }}, 2016$, to December $31^{\text {st }}, 2017$, excluding 16 patients younger than 65 years, 7 patients with conservative treatment, 8 patients with multiple trauma, 2 patients with pathologic fracture, 1 patient with hospital tansfer, 3 patients with in-hospital falls, and 7 patients with no measurement of serum albumin. Finally, 276 patients were included and used as an analysis set of the present study.

Table 1 shows patient characteristics at the enrollment in this study. The patient population $(\mathrm{N}=276)$ were divided into AP negative $(\mathrm{N}=265)$ and AP positive $(\mathrm{N}=11)$ groups. BMI, waiting time, history of hypertension, history of diabetes mellitus, history of circulatory disease, history of respiratory disease, history of renal disease and history of bone fracture showed no significant differences between the groups. On the other hand, age, gender, serum albumin, categorized serum albumin, cognitive impairment and history of osteoporosis showed significant differences between the groups $(p<0.001,0.046,0.033,0.007,0.011$ and $<0.001$, respectively).

Table 2 shows the univariate and multiple logistic regression analysis. Univariate analysis showed that cognitive impairment (OR $12.50,95 \%$ CI 1.09-143, $\mathrm{p}=0.0425$ ) as well as age (OR 1.50, 95\% CI 1.05-2.16, $p=0.0277$ ) were associated with the occurrence of AP. On the other hand, multivariate analysis showed no significant association with the occurrence of AP.

Figure 1 shows the ROC curve of the logistic models, AP positive and AP negative as dichotomous dependent variables, and age, BMI, albumin, cognitive disorder and ADL as independent variables. The area under the curve and its $95 \%$ confidence intervals are as follows: 0.967 (0.893-1.040)

\section{DISCUSSION}

Hip fracture is a common cause of long hospital stay in the 
elderly. In the Japanese practice guideline for treatment of hip fractures suggested that pneumonia is the most common postoperative complication of hip fractures, with an incidence of $3.2 \%$, and the pneumonia is a most common complication of death during hospitalization, accounting for 30 to 44\% [9] through our study showed 11 cases in 276 total cases, i.e., approximately 4\%, previous studies of hip fractures caused by falls have shown rates of complications of pneumonia before and after surgery ranging from 7 to $9 \%$ [10-12] Most evidence guiding perioperative medical risk management of patients undergoing hip fracture repair focuses on cardiac and thromboembolic risk [9], but the present study suggests aspiration pneumonia as one of the important complications for elderly patients after hip fracture repair.

Table 1: Patient characteristics at baseline.

\begin{tabular}{|c|c|c|c|}
\hline Variable & AP Negative $N=265$ & AP Positive $N=11$ & ${ }^{*} p$-value \\
\hline Age (Meant S.D.) & $84.6+7.0$ & $91.8+6.4$ & $<0.001$ \\
\hline Gender, Female: N (\%) & $215(81.1)$ & $6(17.6)$ & 0.046 \\
\hline BM (Meant S.D.) & $21.7+8.5$ & $19.5+4.3$ & 0.425 \\
\hline Waiting Time (Day, Meant S.D.) & $1.7+2.5$ & $1.3+1.2$ & 0.629 \\
\hline Albumin (Meant S.D.) & $3.6+0.6$ & $3.2+0.5$ & 0.033 \\
\hline Albumin, >3.5g/dL: N (\%) & $182(68.7)$ & $3(8.8)$ & 0.007 \\
\hline Cognitive Impairment: N (\%) & $134(50.6)$ & $10(29.4)$ & 0.011 \\
\hline $\begin{array}{c}\text { ADL, Assistance Required: } \mathrm{N} \\
(\%)\end{array}$ & $44(16.6)$ & $4(11.8)$ & 0.104 \\
\hline History of Hypertension N (\%) & $158(59.6)$ & $4(11.8)$ & 0.209 \\
\hline $\begin{array}{l}\text { History of Diabetes Mellitus: N } \\
\qquad(\%)\end{array}$ & $53(20.0)$ & $0(0.0)$ & 0.131 \\
\hline $\begin{array}{l}\text { History of Circulatory Disease: } \\
\qquad \mathrm{N}(\%)\end{array}$ & $75(28.3)$ & $2(5.9)$ & 0.733 \\
\hline $\begin{array}{l}\text { History of Respiratoty Disease: } \\
\qquad \mathrm{N}(\%)\end{array}$ & $32(12.1)$ & $3(8.8)$ & 0.151 \\
\hline History of Renal Disease: N (\%) & $24(9.1)$ & $1(2.9)$ & 1.000 \\
\hline History of Bone Fracture: N (\%) & $85(32.1)$ & 5 (14.7) & 0.346 \\
\hline History of Osteoporosis: N (\%) & $249(94.0)$ & 5 (14.7) & $<0.001$ \\
\hline
\end{tabular}

*t-test (Age, BMI, Waiting time, History of hypertension, History of diabetes mellitus, History of circulatory disease, History of respiratory disease, History of renal disease and History of bone fracture).

Table 2: Results of logistic regression analysis.

\begin{tabular}{|c|c|c|c|c|c|c|c|}
\hline & Coefficients & S.E. & p-value & $\begin{array}{c}\text { Univariate } \\
\text { Odds Ratio }\end{array}$ & 95\% CI & $\begin{array}{c}\text { Adjusted } \\
\text { Odds Ratio }\end{array}$ & $\begin{array}{c}\text { 95\% CI } \\
\text { Age }\end{array}$ \\
\hline BMI & 0.807 & 0.445 & 0.070 & 1.502 & $1.046-2159$ & 2.242 & $0.937-5.367$ \\
\hline Albumin & 0.056 & 0.200 & 0.779 & 0.839 & $0.632-1.114$ & 1.058 & $0.714-1.567$ \\
\hline Cognitive & 0.425 & 1.060 & 0.688 & 0.821 & $0.291-2.316$ & 1.530 & $0.192-12.213$ \\
\hline ADL & -3.125 & 4.115 & 0.137 & 12,500 & $1.089-143.4$ & 457.337 & $0.144-1000$ \\
\hline
\end{tabular}

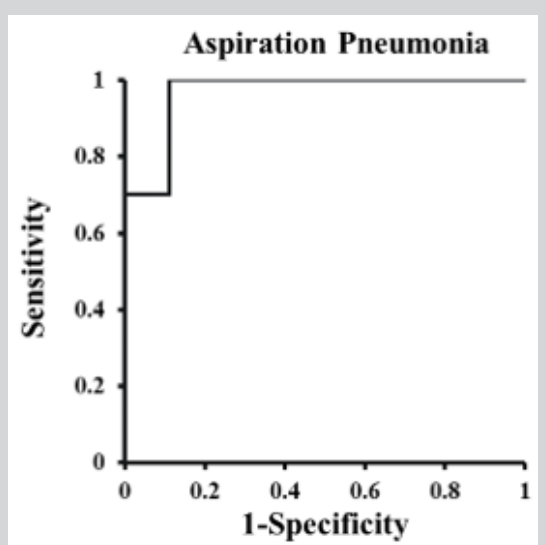

Figure 1: ROC curves of the logistic regression model.
The present retrospective study has suggested that the age, gender, serum albumin and cognitive impairment could be risk factors for AP in patients receiving post-operative surgery of femoral neck and trochanteric fractures.

Regarding the patient background, as shown in Table 1, age, gender, serum albumin, categorized serum albumin, cognitive impairment and history of osteoporosis showed significant differences between the AP positive and AP negative groups ( $p$ $<0.001,0.046,0.033,0.007,0.011$ and $<0.001$, respectively). The results suggested that age, gender, serum albumin and cognitive impairment were associated with AP occurrence. On the other hand, no significant differences were observed in the BMI, waiting time, ADL, history of hypertension, history of diabetes mellitus, history of circulatory disease, history of respiratory disease, history of renal disease and history of bone fracture, indicating that the AP 
occurrence is less associated with history of such diseases. The results of the multiple logistic regression analyses were shown in Table 2, which suggested that serum albumin, cognitive impairment and ADL were not associated with the occurrence of AP under adjustment of age and BMI as covariates using appropriate logistic model (ROC_AUC=0.967).

The present study showed association of serum albumin level with occurrence with AP in the patient background. Consistent with our results, Metani et al. [15], also reported significant reduction in serum albumin in AP cases. They also indicated lower $\mathrm{BMI}$ in AP cases, indicating that patients with low BMI, including low nutritional cases, can have lower resistance to infection. The calculation of the cut-off value by future research is meaningful from the viewpoint of the prevention of the pneumonia.

Complications of pneumonia do not necessarily directly lead to death, but they are anticipated to result in decreased motor function and other physical activity. Aspiration pneumonitis associated with cerebrovascular accident has been reported to result in a marked decrease in Barthel Index and Modified Rankin scale [18]. Although the present study is a retrospective research, it could provide insight with respect to aspiration pneumonia as a risk factor in patients with hip fracture. And based on the results of this study, we treat perioperative complications under the multidisciplinary cooperation and improve the quality of life of elderly patients after discharge $[19,20]$. Future investigations into the association between aspiration pneumonia and physical activity will be expected.

\section{CONCLUSION}

In conclusion, we retrospectively evaluated the risk factors for AP in patients with femoral neck and trochanteric fractures, showed that serum albumin and cognitive function seemed to be the weak risk factors for AP but were necessary to assess under adjustment of confounding factors, including age, gender and BMI. Assistance of cognitive function seemed to be important for the postoperative management of AP, specifically in elderly patients receiving surgery of femoral neck and trochanteric fractures.

\section{REFERENCES}

1. Carneiro MB, Alves DPL, Mercadante MT (2013) Physical therapy in the postoperative of proximal femur fracture in elderly. Literature review. Acta Ortopedica Brasileira 21: 175-178.

2. Gregersen M, Morch MM, Hougaard K, Damsgaard EM (2012) Geriatric intervention in elderly patients with hip fracture in an orthopedic ward. J Inj Violence Res 4: 45-51.

3. Hagino H, Endo N, Harada A (2017) Survey of hip fractures in Japan: Recent trends in prevalence and treatment. J Orthop Sci 22: 909-914.

4. http://wwwcitytoyamatoyamajp/kikakukanribu/johotokeika/tokei

5. Avenell A, Smith TO, Curtain JP, Mak JC, Myint PK, et al. (2016) Nutritional supplementation for hip fracture aftercare in older people. Cochrane Database Syst Rev 11: Cd001880.
6. Chang SC, Lai JI, Lu MC (2018) Reduction in the incidence of pneumonia in elderly patients after hip fracture surgery: An inpatient pulmonary rehabilitation program. Medicine (Baltimore) 97: e11845.

7. Chotanaphuti T, Jareonarpornwatana A, Laoruengthana A (2009) The mortality rate after thromboembolism prophylaxis in the hip fracture surgery. J Med Assoc Thai 92 Suppl 6: S115-119.

8. Chughtai M, Gwam CU, Mohamed N, Khlopas A, Jared M, et al. (2017) The Epidemiology and Risk Factors for Postoperative Pneumonia. J Clin Med Res 9: 466-475.

9. Lawrence VA, Hilsenbeck SG, Noveck H, Poses RM, Carson JL, et al. (2002) Medical complications and outcomes after hip fracture repair. Arch Intern Med 162: 2053-2057.

10. Khan MA, Hossain FS, Ahmed I, Muthukumar N, Mohsen A (2013) Predictors of early mortality after hip fracture surgery. Int Orthop 37: 2119-2124.

11. Radcliff TA, Henderson WG, Stoner TJ, Khuri SF, Dohm M, et al. (2008) Patient risk factors, operative care, and outcomes among older community-dwelling male veterans with hip fracture. J Bone Joint Surg Am 90: 34-42.

12. Roche JJ, Wenn RT, Sahota O, Moran CG (2005) Effect of comorbidities and postoperative complications on mortality after hip fracture in elderly people: Prospective Observational Cohort Study. Bmj 331: 1374.

13. Ganczak M, Chrobrowski K, Korzen M (2018) Predictors of a Change and Correlation in Activities of Daily Living after Hip Fracture in Elderly Patients in a Community Hospital in Poland: A Six-Month Prospective Cohort Study. Int J Environ Res Public Health 15.

14. Higashikawa T, Shigemoto K, Goshima K, Usuda D (2020) Risk factors for the development of aspiration pneumonia in elderly patients with femoral neck and trochanteric fractures: A Retrospective Study of a Patient Cohortmedicine (Baltimore) 99(7): E19108.

15. Metani H, Tsubahara A, Hiraoka T, Seki S, Hasegawa T (2015) Risk factors for patients who develop pneumonia either before or after hip fracture surgery. Japanese Journal of Comprehensive Rehabilitation Science 6: 43-49.

16. Kanda Y (2013) Investigation of the freely available easy-to-use software 'EZR' for medical statistics. Bone Marrow Transplant 48: 452-458.

17. The Japanese orthopaedic association (2015) Japanese society for fracture repair. Practice guideline for treatment of femoral neck and trochanteric fractures Tokyo, Japan.

18. Aslanyan S, Weir CJ, Diener HC, Kaste M, Lees KR (2004) Pneumonia and urinary tract infection after acute ischaemic stroke: A Tertiary Analysis of the GAIN International Trial. Eur J Neurol 11: 49-53.

19. Higashikawa T, Shigemoto K, Goshima K, Usuda D (2019) Urinary retention as a postoperative complication associated with functional decline in elderly female patients with femoral neck and trochanteric fractures: A Retrospective Study of a Patient Cohort. Medicine (Baltimore) 98(24): e16023.

20. Higashikawa T, Shigemoto K, Goshima K, Horii T Mortality (2020) Risk factors in elderly female patients with femoral neck and trochanteric fractures. J Clin Med Res 12(10): 668-673. 